mind, I arrive at a galactic neutron star birth rate of 1 in $20 \pm 10 \mathrm{yr}$.

\section{W. KUNDT}

Institut für Astrophysik, Universität Bonn,5300 Bonn, FRG

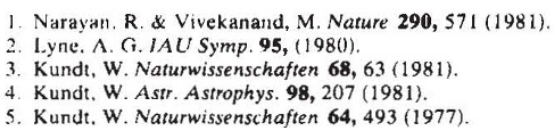

NARAYAN AND VIVEKANAND REPLY-It is known' that the radio luminosities of pulsars decrease with age. Therefore, the observed sample of pulsars is biased towards the more luminous younger pulsars. Consequently, the average age of pulsars determined ${ }^{2}$ from the observed fraction of young pulsars $\left(\tau<10^{6} \mathrm{yr}\right)$ underestimates the true mean pulsar age. The bias is probably even more stronger in the kinematic ages determined from $z / \dot{z}$ because proper motions have been reliably obtained only for relatively luninous pulsars. On the other hand, in our analysis, we have accounted for radio luminosity selection effects and thereby obtained an approximation to the complete galactic population of pulsars. Consequently, our result $N / N=11 \times 10^{6}$ $\mathrm{yr}$ is unbiased and a more reliable estimate of the mean pulsar age than the earlier approaches which typically gave $4 \times 10^{6}$ yr. Note, however, that age is not a very relevant parameter in our analysis, which is based entirely on pulsar flow in the $P-\dot{P}$ diagram (pulsar period to its time derivative). Moreover, the concept of mean pulsar age itself may not be very meaningful or useful if pulsars belong to more than one class with widely different active lifetimes.

Regarding the beaming factor $K$, we consider that the $5 \%$ occurrence of interpulses is consistent with a circular beam cross section and a value of $K=5$ rather than with banana-shaped cross sections and $K=1.0$

\section{RAMESH NARAYAN M. VIVEKANAND \\ Raman Research Institute, Bangalore 560080 India}

1. A. G. Lyne, R. T. Ritchings \& F. G. Smith Mon. Not. R. astr. SOC. 171, 579-597 (1975).

2. A. G. Lyne. IAU Symp. 95, (1980).

\section{Solar-flare produced ${ }^{3} \mathrm{He}$ in lunar samples}

RAO AND VENKATESAN ${ }^{1}$ have reported a method for deducing the absolute solar cosmic ray (SCR) proton fluxes in the past few million years using SCR protoninduced ${ }^{3} \mathrm{He}$ in the top surface layers of Moon rocks. To quote the authors: "even if there are minor diffusion losses, this method holds good as long as the losses are not depth dependent". I would like to point out that Moon rocks studied in this laboratory which are similar to those used by Rao and Venkatesan show severe ${ }^{3} \mathrm{He}$ diffusion losses, up to $99 \%$ (refs 2-6), and the losses show strong depth dependence $^{3-6}$. Another problem arises from the directly implanted solar flare ${ }^{3} \mathrm{He}$ found in the top few millimetres of these Moon rocks ${ }^{3-6}$. This ${ }^{3} \mathrm{He}$ component shows depth dependence and is about one order of magnitude higher in concentration than the SCR proton-induced ${ }^{3} \mathrm{He}$, therefore masking it in the top few millimetres of rock ${ }^{3}$. The rocks which we have studied, 68815 and 65315, were excavated from south ray crater, have GCR and SCR exposure ages of $2 \mathrm{Myr}$, contain plagioclase as the major mineral and therefore are similar in many characteristics to the rocks studied by Rao and Venkatesan, 61016 and 64435. The near constancy of ${ }^{3} \mathrm{He} /{ }^{21} \mathrm{Ne}$ ratios which they suggest as an indication that depth dependence diffusion effects are not significant can also be misleading as the ${ }^{2}$ Ne diffusion pattern shows in our rocks a similar depth dependence to the ${ }^{3} \mathrm{He}$ and therefore the ${ }^{3} \mathrm{He} /{ }^{21} \mathrm{Ne}$ ratio might stay quite constant. We believe that Rao and Venkatesan were unable to observe the two effects, depth dependence diffusion and the directly solar flare-implanted ${ }^{3} \mathrm{He}$ because first, only three layers of different depths were taken from each rock, and second, the layers were too thick $(\geqslant 1.5 \mathrm{~mm})$. We can judge from our fine sampling, nine for each rock in the range $0-15 \mathrm{~mm}$ and $\sim 0.2 \mathrm{~mm}$ thick layers in the first $0-1 \mathrm{~mm}$ of range, that neither the diffusion pattern nor the SCR-implanted ${ }^{3} \mathrm{He}$ would have been observed using the sampling technique of Rao and Venkatesan. I therefore consider ${ }^{3} \mathrm{He}$ to be the wrong isotope for the use proposed unless the effects mentioned are taken into account.

\section{Department of Physics and Astronomy}

Tel Aviv University,

Ramat Aviv, Israel

$$
\begin{aligned}
& \text { 1. Rao, M. N. \& Venkatesan. T.R. Nature 286, 788-790 } \\
& \text { (1980). } \\
& \text { 3. Yaniv, A. \& Marti, K. Meteoritics 15, } 390 \text { (1980). } \\
& \text { 4. Yaniv, A. \& Marti, K. Astr. Phys. J. Lett. 247, L1-1.4 } \\
& \text { (1981). } \\
& \text { 5. Yaniv, A., Kirsten, T. \& Richter, H. Astr. Phys. J. Lett. } \\
& \text { (submitted). } \\
& \text { 6. Yaniv, A. \& Kirsten, T. Proc. 12th lunar planet. Sci. Conf., } \\
& \text { 1224-1226 (1981). }
\end{aligned}
$$

RAO AND VENKATESAN REPLY -We point out that we discussed these issues in detail elsewhere ${ }^{1}$. Relevant points are briefly outlined below.

Our inferences regarding ${ }^{3} \mathrm{He}$ losses are based on experimental results in rocks
61016 and 64435 . The general agreement (within a factor of two) of exposure ages based on SCR-produced ${ }^{21} \mathrm{Ne}$ and ${ }^{38} \mathrm{Ar}$ and ${ }^{3} \mathrm{He}$, with the surface exposure ages based on particle tracks, could not have been observed if almost total loss of ${ }^{3} \mathrm{He}$ had taken place by diffusion in these samples. Further, the diffusion effects of ${ }^{3} \mathrm{He}$ and ${ }^{21} \mathrm{Ne}$ are mass-dependent. Yaniv may not be observing such mass-dependent effects because $99 \%$ of the gas has been lost from his samples. The depthdependent diffusion will lead to variations in the ${ }^{3} \mathrm{He} /{ }^{21} \mathrm{Ne}$ and ${ }^{3} \mathrm{He} /{ }^{38} \mathrm{Ar}$ ratios with depth, which is not borne out by the nearuniformity of these observed ratios in our samples. (Details are discussed in refs 1 , 2.)

The peak temperatures to which some of these rocks were exposed on the lunar surface is $\sim 370 \mathrm{~K}$ and the average temperature is $210 \mathrm{~K}$; He loss can be partial but not almost total, at such temperatures, in these samples.

In some lunar plagioclases, ${ }^{3} \mathrm{He}$ was lost and in some it was well retained. In a sensitive Gas ion-probe analysis of ${ }^{4} \mathrm{He}$ in plagioclases, Müller et al. observed large amounts of ${ }^{4} \mathrm{He}$ in two plagioclases (P4 and P2), with a capability of retention similar to that of ilmenite, whereas in some other plagioclases from soil 76501 , no ${ }^{4} \mathrm{He}$ was found. Shock effects also seem to be involved.

The occurrence and composition of directly implanted solar flare $\mathrm{He}$ and $\mathrm{Ne}$ in lunar samples is important. We are investigating the composition of solar flare $\mathrm{Ne}$ in these samples by selective chemical etching and subsequent stepwise thermal release methods. The long-term solar flare $\mathrm{Ne}$ composition seems to be similar to fractionated solar wind ${ }^{4}$. The long-term solar flare $\mathrm{He}$ composition is not well known, but the contemporary solar flare ${ }^{3} \mathrm{He} /{ }^{4} \mathrm{He}$ ratios (measured from spacecraft) vary from 1 to 0.01 and differs between flares ${ }^{5}$. Most of the ${ }^{3} \mathrm{He}$-rich flares are very weak and their contribution to the total He seems to be limited. It is not clear whether the long-term solar flare $\mathrm{He}$ composition is similar to the fractionated solar wind as in the case of $\mathrm{Ne}$ or varies drastically as observed in contemporary flare studies. Further controlled experiments are under way to understand these complexities.

\section{N. RAO \\ T. R. VENKATESAN}

Physical Research Laboratory.

Navrangpura,

Ahmedabad 380009 .

India

1. Venkatesan, T. R. et al. Proc. It th lunar planet. Sci. Conf. 1271-1284 (1980).

2. Rao. M. N. \& Venkatesan, T. R. Nature 286, 788-790 (1980).

3. Müller, H. W. et al. Proc. 7th lunar Sci. Conf. 937-951 (1976)

4. Nautiyal, C. M. et al. Proc. 12 th lunar planet. Sci. Conf. (1981) (in the press).

Ramaty R. et al.NASA. Tech. Rep. 79660 (1978) 